\title{
Synthesizing a Multiband LTCC Bandpass Filter With Specified Transmission- and Reflection-Zero Frequencies
}

\author{
Yi-Chieh Lin, Student Member, IEEE, Tzyy-Sheng Horng, Senior Member, IEEE, and Hui-Hsiang Huang
}

\begin{abstract}
This paper presents a vertically expandable low-temperature cofired ceramic (LTCC) bandpass filter (BPF) structure to increase the number of operating bands of the BPF without changing the area of the footprint. To design efficiently the proposed LTCC BPFs with highly configurable passbands and stopbands, a new method of synthesizing multiband BPF prototypes with specified transmission- and reflection-zero frequencies is developed. A dual-band BPF and a triple-band BPF were designed and implemented in LTCC. Both BPFs have the same area of $1.25 \times 2.0 \mathrm{~mm}^{2}$. Comparisons of the $S$-parameters among the synthesized prototypes, postlayout simulations and measurements exhibit a strong correlation.
\end{abstract}

Index Terms-Filter synthesis method, low-temperature cofired ceramic (LTCC) bandpass filters (BPFs), multiband bandpass filters, reflection zeros, transmission zeros.

\section{INTRODUCTION}

M ICROWAVE bandpass filters (BPFs) are essential components in radio frequency (RF) front-ends of wireless communication systems that reject unwanted spurious and interference signals. Low-temperature cofired ceramic (LTCC) BPFs have an important role in these systems because they provide satisfactory performance with a compact size and reasonable cost. However, the trend toward multiband multimode mobile terminals requires smaller and more highly integrated LTCC BPFs [1]. Therefore, designing miniaturized LTCC multiband BPFs is becoming increasingly challenging. Although the counterpart printed circuit board (PCB) BPF designs have achieved up to a hexa-band performance [2], [3], and employed a stacked structure to further reduce the occupied area [4], the issue of component size still remains to be of major concern.

To fulfill the multiband operation for RF front-ends, LTCC diplexers and multiplexers are often utilized to separate signals

Manuscript received July 01, 2014; revised August 22, 2014; accepted October 08,2014 . Date of publication November 20, 2014; date of current version December 02, 2014. This work was supported in part by the Ministry of Science and Technology, Taiwan, under Grant 101-2622-E-110-005-CC3, Grant 100-2221-E-110-081-MY3, and Grant 100-2221-E-110-082-MY3. This paper is an expanded version from the IEEE MTT-S International Microwave Symposium, Tampa Bay, FL, USA, June 1-6, 2014.

Y.-C. Lin and T.-S. Horng are with the Department of Electrical Engineering, National Sun Yat-Sen University, Kaohsiung 804, Taiwan (e-mail: jason@ee. nsysu.edu.tw).

H.-H. Huang is with the INFOMAX Communication Company Ltd., Hsinchu 300, Taiwan.

Color versions of one or more of the figures in this paper are available online at http://ieeexplore.ieee.org.

Digital Object Identifier 10.1109/TMTT.2014.2365454 with different carrier frequencies [5]-[8], but these components are usually used with many other single-band BPFs and narrowband active circuits in an RF front-end, leading to poor system integration. In contrast, multiband LTCC BPFs along with wideband active circuits allow greater integration and favor a less complex RF front-end. Dual-band [9]-[16] and tri-band [17], [18] LTCC BPFs have been explored extensively in the literature. Combining two single-band LTCC BPFs into one dual-band LTCC BPF is relatively straightforward, as demonstrated in [9] and [10]. However, the merging of more than two single-band LTCC BPFs is not very viable because of the complicated impedance matching issues that arise. Coupled semilumped [11], [12] and stepped-impedance [13], [14] resonator structures are popular in dual-band LTCC BPF designs. Nevertheless, increasing the number of operating bands is difficult owing to limitations on the coupling mechanism or the microstrip impedance control. Ridge waveguide [15] and substrate integrated waveguide [16], [17] filters in LTCC increase the design flexibility for multiband BPFs by exciting and coupling cavity resonators. However, these components are usually too large for commercial use in the main mobile terminals and need more complicated cavity resonator structures such as capacitively loaded cavities [19], [20] for further miniaturization.

LTCC BPFs in previous studies are not only miniature, but also exploit transmission zeros to enhance their passband selectivity and stopband attenuation. Common methods for creating transmission zeros in LTCC BPFs include adding inductive, capacitive or transmission-line elements to the resonators [21]-[23], introducing extra electrical or magnetic coupling between the resonators [21], [24] and incorporating grounding inductors and capacitors [25]-[27]. Although quite effective, a shortcoming of these methods is that the transmission-zero frequencies are not determined independently of each other, making the optimization of multiple transmission zeros to maximize attenuation at the targeted stopband frequencies an arduous task. In addition, the previous studies in [28] and [29] use reflection zeros to determine the passband frequency range of single-band LTCC BPFs. A recent work by the present authors extends the use of reflection zeros to multiband LTCC BPFs [30].

Our work in [30] synthesized a dual-band LTCC BPF with the designated transmission- and reflection-zero frequencies. Although the key equations for the synthesis were derived, no specific method for solving these partly nonlinear equations was 


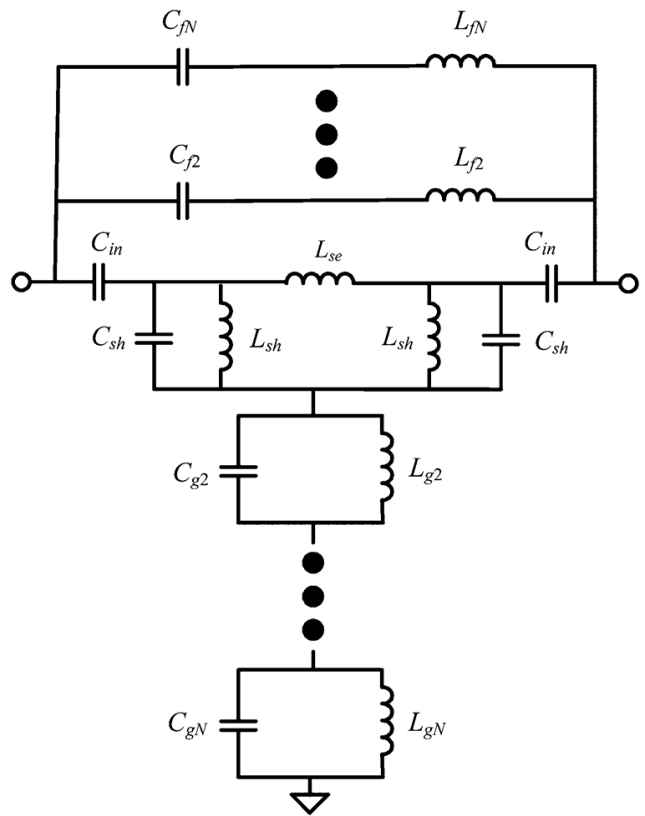

Fig. 1. Proposed topology of the vertically expandable multiband LTCC BPFs.

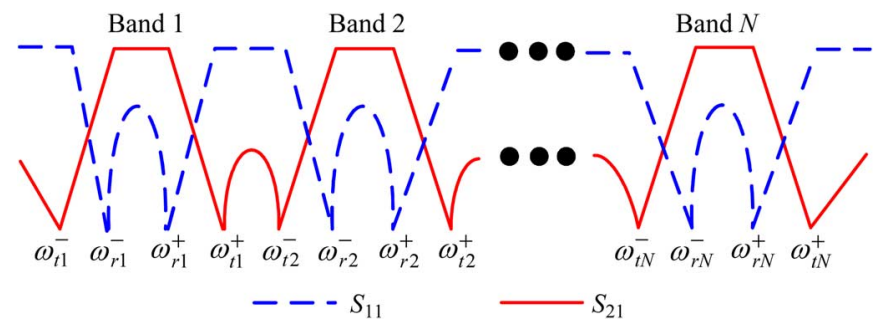

Fig. 2. Schematic diagram of the multiband BPF response configured with the transmission-zero and reflection-zero frequencies.

established. This work provides a hybrid procedure to solve the linear transmission-zero equations and nonlinear reflection-zero equations associated with this synthesis problem. Furthermore, an additional tri-band LTCC BPF is realized to confirm the robustness of the developed synthesis procedure.

\section{Filter TOPOLOGY}

The proposed multiband BPF is based on a single-band BPF that uses a second-order inductively coupled resonator (ICR) network. As shown in Fig, 1, the filter topology comprises series-connected shunt $L C$ resonators between the ICR network and the ground, and parallel-connected series $L C$ resonators between the input and the output of the ICR network. The multistage $L_{g n} C_{g n}$ and $L_{f n} C_{f n}$ resonant circuits, $n=2,3, \ldots, N$, form the series and parallel feedback network, respectively, to create $N-1$ additional passbands. Fig. 2 presents a schematic frequency response of this filter topology. In this figure, $\omega_{r i}^{-}$and $\omega_{r i}^{+}, i=1,2, \ldots, N$, are the reflection-zero frequencies, while $\omega_{t i}^{-}$and $\omega_{t i}^{+}, i=1,2, \ldots, N$, are the transmission-zero frequencies. A particular feature is two reflection zeros in each passband and two transmission zeros between adjacent passbands. The proposed filter topology is very suitable for implementation using multilayer LTCC technology because the required area is the same as that for an ICR network and the height increases with the number of layers of the two feedback resonant

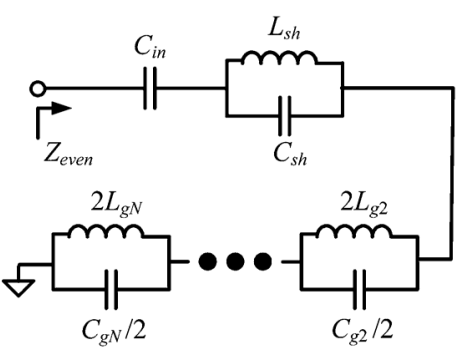

(a)

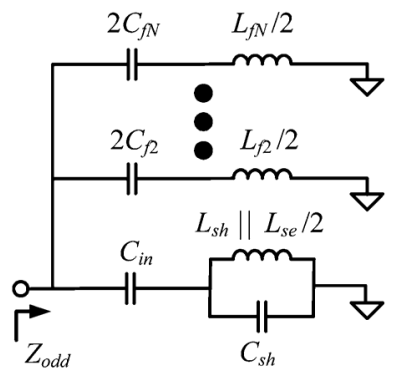

(b)
Fig. 3. Decomposition of the proposed BPF topology into two half circuits. (a) Even-mode half circuit. (b) Odd-mode half circuit.

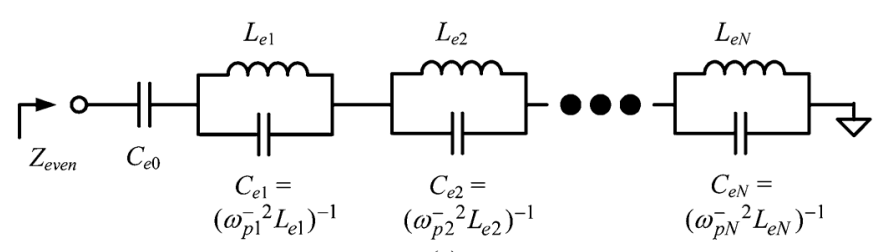

(a)

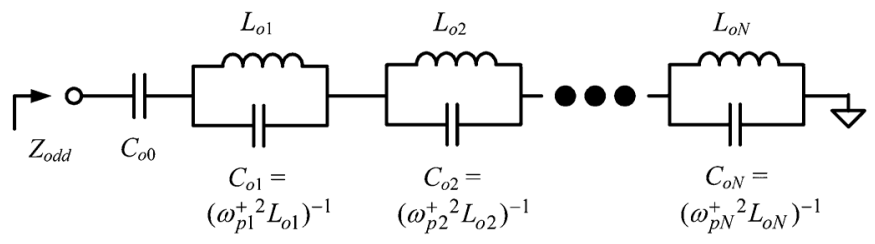

(b)

Fig. 4. Foster series equivalent circuits of the even-mode and odd-mode impedances. (a) Even-mode half circuit. (b) Odd-mode half circuit.

networks. Accordingly, this filter topology is useful for miniaturizing LTCC multiband BPFs with a strict area constraint.

Symmetry can be utilized to decompose the filter topology that is shown in Fig. 1 into odd-mode and even-mode half circuits, as displayed in Fig. 3(a) and (b), respectively. The oddmode half circuit in Fig. 3(b) has a series Foster equivalent circuit with the same topology as that of the even-mode half circuit, as illustrated in Fig. 4(a) and (b). The input impedances of these half circuits are expressed as

$$
\begin{aligned}
Z_{\text {even }}(\omega)= & \frac{1}{j \omega C_{i n}}+j \omega L_{s h}\left\|\frac{1}{j \omega C_{s h}}+\sum_{n=2}^{N} j 2 \omega L_{g n}\right\| \frac{2}{j \omega C_{g n}} \\
= & \frac{1}{j \omega C_{e 0}}+\sum_{n=1}^{N} \frac{j \omega L_{e n}}{1-\left(\frac{\omega}{\omega_{p n}^{-}}\right)^{2}} \\
Z_{\text {odd }}(\omega)= & {\left[\left(\frac{1}{j \omega C_{i n}}+j \omega L_{s h}\left\|j \omega \frac{L_{s e}}{2}\right\| \frac{1}{j \omega C_{s h}}\right)^{-1}\right.} \\
& \left.+\sum_{n=2}^{N}\left(\frac{j \omega L_{f n}}{2}+\frac{1}{j 2 \omega C_{f n}}\right)^{-1}\right]^{-1} \\
= & \frac{1}{j \omega C_{o 0}}+\sum_{n=1}^{N} \frac{j \omega L_{o n}}{1-\left(\frac{\omega}{\omega_{p n}^{+}}\right)^{2}}
\end{aligned}
$$

where $\omega_{p n}^{-}$and $\omega_{p n}^{+}, n=1,2, \ldots, N$, are the resonant pole frequencies of the even-mode and odd-mode half circuit, respectively. The circuit in Fig. 4(b) can be transformed to that in 
Fig. 3(b) by performing a pole-residue analysis and their conversion equations are as follows:

$$
\begin{aligned}
L_{f n}=\frac{1}{R_{n}}, & n=2,3, \ldots, N \\
C_{f n}=\frac{R_{n}}{\omega_{q n}^{2}}, & n=2,3, \ldots, N
\end{aligned}
$$

where

$$
R_{n}=\lim _{s \rightarrow j \omega_{q n}} Y_{\text {odd }}(s)\left(s-j \omega_{q n}\right)
$$

are the residues that correspond to the poles $s=j \omega_{q n}$ of the odd-mode input admittance $Y_{\text {odd }}(s)=1 / Z_{\text {odd }}(s)$. The remaining circuit elements are determined as

$$
\begin{aligned}
C_{i n} & =C_{o 0}-2 \sum_{n=2}^{N} C_{f_{n}} \\
C_{s h} & =\left(\sum_{n=0}^{N} \frac{1}{C_{o n}}-\frac{1}{C_{i n}}\right)^{-1} \\
L_{s h} \| \frac{L_{s e}}{2} & =\frac{1}{\omega_{q 1}^{2}\left(C_{i n}+C_{s h}\right)}
\end{aligned}
$$

where $\omega_{q 1}$ is the fundamental pole frequency that results from the core ICR network. The impedance parameters of the filter topology, shown in Fig. 1, can be derived in terms of the evenand odd-mode input impedances, as follows:

$$
\begin{aligned}
& Z_{11}(\omega)=Z_{22}(\omega)=\frac{\left(Z_{\text {even }}(\omega)+Z_{\text {odd }}(\omega)\right)}{2} \\
& Z_{12}(\omega)=Z_{21}(\omega)=\frac{\left(Z_{\text {even }}(\omega)-Z_{\text {odd }}(\omega)\right)}{2} .
\end{aligned}
$$

The $S$-parameters are further obtained from the impedance parameters as

$$
\begin{aligned}
& S_{12}(\omega)=S_{21}(\omega)=\frac{\left(Z_{\text {even }}(\omega)-Z_{\text {odd }}(\omega)\right) Z_{0}}{\left(Z_{\text {even }}(\omega)+Z_{0}\right)\left(Z_{\text {odd }}(\omega)+Z_{0}\right)} \\
& S_{11}(\omega)=S_{22}(\omega)=\frac{Z_{\text {even }}(\omega) Z_{\text {odd }}(\omega)-Z_{0}^{2}}{\left(Z_{\text {even }}(\omega)+Z_{0}\right)\left(Z_{\text {odd }}(\omega)+Z_{0}\right)} .
\end{aligned}
$$

The transmission- and reflection-zero frequencies can be determined by setting (11) and (12) to zero, respectively, yielding the following equations.

$$
\begin{aligned}
& \left\{\begin{array}{l}
Z_{\text {even }}\left(\omega_{t i}^{-}\right)=Z_{\text {odd }}\left(\omega_{t i}^{-}\right) \\
Z_{\text {even }}\left(\omega_{t i}^{+}\right)=Z_{\text {odd }}\left(\omega_{t i}^{+}\right)
\end{array}, \quad i=1,2, \ldots, N\right. \\
& \left\{\begin{array}{l}
Z_{\text {even }}\left(\omega_{r i}^{-}\right) Z_{\text {odd }}\left(\omega_{r i}^{-}\right)=Z_{0}^{2} \\
Z_{\text {even }}\left(\omega_{r i}^{+}\right) Z_{\text {odd }}\left(\omega_{r i}^{+}\right)=Z_{0}^{2}
\end{array}, \quad i=1,2, \ldots, N .\right.
\end{aligned}
$$

In the design of a bandpass filter with $N$ passbands, the filter response can be constructed by designating an appropriate set of $2 N$ reflection zeros and $2 N$ transmission zeros, as revealed by Fig. 2. Therefore, (13) and (14) form a set of $4 N$ equations with these transmission- and reflection-zero frequencies that are

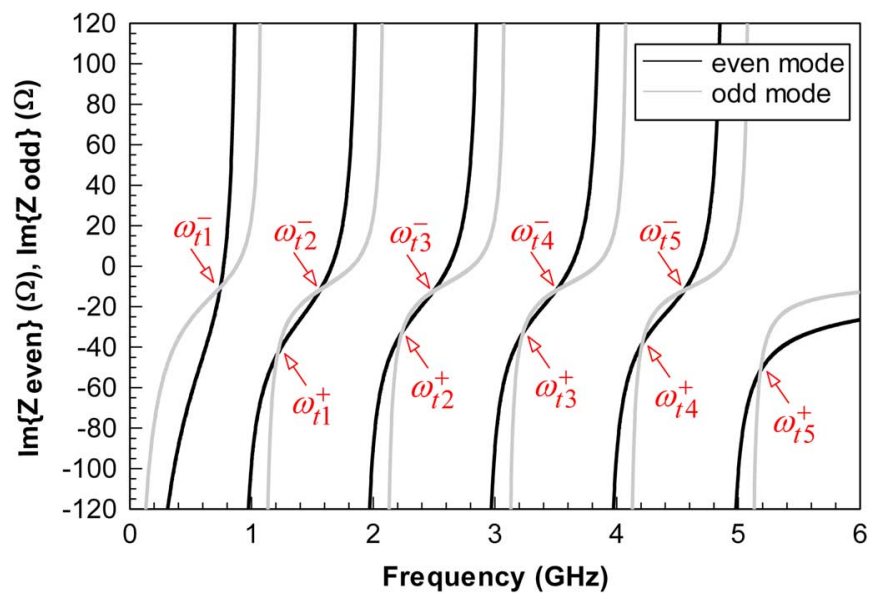

Fig. 5. Illustration of the creation of transmission zeros using the even-mode and odd-mode impedances with staggered resonant pole frequencies.

solved for the $4 N$ unknown elements in the topology of Fig. 1. In Fig. 1, the core ICR network has four elements $L_{s e}, C_{i n}, L_{s h}$, and $C_{s h}$ and the $N-1$ pairs of $L_{g n} C_{g n}$ and $L_{f n} C_{f n}$ resonators have a total of $4(N-1)$ elements. Importantly, the consideration of insertion and return loss levels needs to be included in the assignment of transmission- and reflection-zero frequencies at the beginning of the design procedure. Below are some guidelines.

1) In the passband, the closer the two adjacent reflection-zero frequencies are, the lower the insertion-loss level or the higher the return-loss level between them.

2) In the stopband, the closer the two adjacent transmissionzero frequencies are, the higher the rejection level between them.

3) In the transition from passband to stopband, the closer the adjacent transmission- and reflection-zero frequencies, the sharper the rolloff between them.

The equivalent half circuits that are shown in Fig. 4(a) and (b) can be utilized to configure quickly a multiband BPF prototype. The main idea is to establish the even-mode and odd-mode half circuits with staggered resonant pole frequencies. For example, to configure a penta-band BPF prototype with passbands within the frequency ranges of 0.9-1.1, 1.9-2.1, 2.9-3.1, 3.9-4.1, and 4.9-5.1 GHz, both used half circuits are five stages $(N=5)$ with the following sets of even-mode and odd-mode resonant pole frequencies; $\left(\omega_{p 1}^{-}, \omega_{p 2}^{-}, \omega_{p 3}^{-}, \omega_{p 4}^{-}, \omega_{p 5}^{-}\right)=2 \pi \times(0.9 \mathrm{GHz}, 1.9 \mathrm{GHz}, 2.9 \mathrm{GHz}$, $3.9 \mathrm{GHz}, 4.9 \mathrm{GHz})$, and $\left(\omega_{p 1}^{+}, \omega_{p 2}^{+}, \omega_{p 3}^{+}, \omega_{p 4}^{+}, \omega_{p 5}^{+}\right)=2 \pi \times$ $(1.1 \mathrm{GHz}, 2.1 \mathrm{GHz}, 3.1 \mathrm{GHz}, 4.1 \mathrm{GHz}, 5.1 \mathrm{GHz})$. Then, from experimental experience, the following circuit element values are selected; $C_{e 0}=4 \mathrm{pF}, C_{o 0}=10 \mathrm{pF}, L_{e n}=2.5 / n^{2} \mathrm{nH}$, and $L_{o n}=1 / n^{2} \mathrm{nH}, n=1,2,3,4,5$. Given the above values of the variables in (1) and (2), the frequency responses of the even-mode and odd-mode impedances $Z_{\text {even }}(\omega)$ and $Z_{\text {odd }}(\omega)$ are calculated, as displayed in Fig. 5, where arrows point to the intersections of these two responses. According to (13), the frequencies at these intersections are the transmission-zero frequencies with values of $0.744,1.215,1.566$, $2.235,2.494,3.229,3.5,4.211,4.557$, and $5.182 \mathrm{GHz}$. Fig. 6 plots the product of the even- and odd-mode impedances 


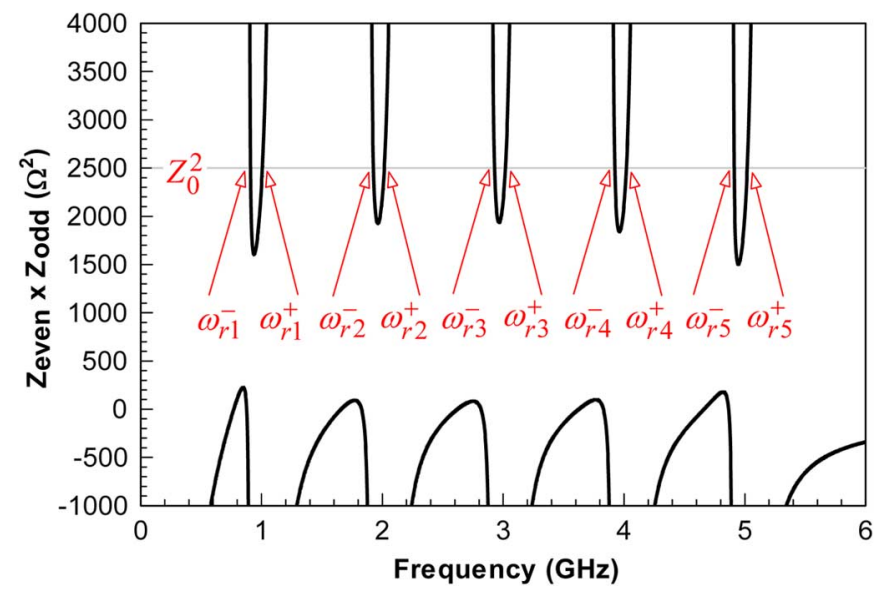

Fig. 6. Illustration of the creation of reflection zeros using the product of the even-mode and odd-mode impedances.

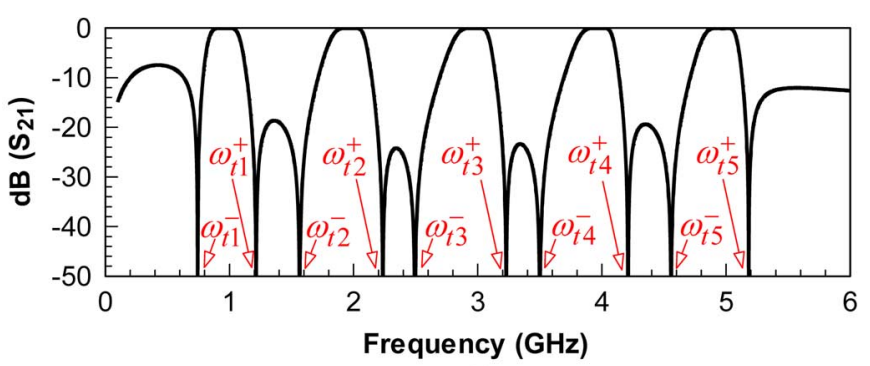

(a)

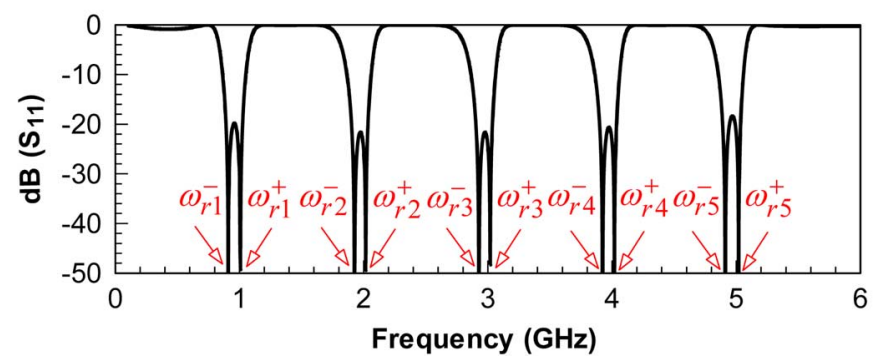

(b)

Fig. 7. Composition of a penta-band BPF response with the even-mode and odd-mode impedances shown in Figs. 5 and 6. (a) Magnitude of $S_{21}$. (b) Magnitude of $S_{11}$.

$Z_{\text {even }}(\omega) \times Z_{\text {odd }}(\omega)$, with arrows that point to the positions at which $Z_{\text {even }}(\omega) \times Z_{\text {odd }}(\omega)=Z_{0}^{2}=2500 \Omega$. According to (14), the frequencies at these positions are necessarily the reflection-zero frequencies, which are $0.91,1.007,1.927$, 2.017, 2.929, 3.019, 3.924, 4.02, 4.912, and 5.02 GHz. These reflection-zero frequencies will be closer to the resonant pole frequencies when a higher system impedance $\left(Z_{0}\right)$ is used. Fig. 7(a) and (b) plot the computed magnitudes of $S_{21}$ and $S_{11}$ using (11) and (12), respectively. These two figures verify the frequencies of the transmission and reflection zeros that are obtained by analysis of Figs. 5 and 6 .

The above example elucidates how the proposed filter topology functions for a multiband BPF but not how to determine the element values of the multiband BPF, because the above example fails to address two important issues. The first issue is the means of ensuring that the transmission and reflection zeros are at the specified frequencies. This imposition requires a simultaneous solution to the two sets of equations,
(13) and (14), of which the former is linear but the latter is nonlinear. The other issue concerns ensuring the same values for the common elements in the even-mode and odd-mode half circuits of Fig. 3(a) and (b). These common elements are $C_{i n}, C_{s h}$ and $L_{s h}$; the inductance $L_{s h}$ does not pose a problem because it is in parallel with another inductance $L_{s e}$ in the odd-mode half circuit, so it can be varied as long as the inductances $L_{s h}$ and $L_{s e}$ in parallel satisfy (8). To overcome the two aforementioned issues, a procedure was developed for synthesizing a multiband BPF prototype with the specified transmission- and reflection-zero frequencies while meeting the consistency requirements in determining $C_{i n}$ and $C_{s h}$. The following section will discuss this procedure in detail.

\section{SyNTHesis PRocedURE}

This section describes a hybrid analytical and iterative procedure for solving the two sets of (13) and (14) to implement the multiband BPF design with the specified transmission- and reflection-zero frequencies. With reference again to Figs. 3(b) and 4(b), the equivalence of the two odd-mode half circuits yields the following equation as the frequency approaches infinity,

$$
\frac{1}{C_{i n}}+\frac{1}{C_{s h}}=\frac{1}{C_{o 0}}+\sum_{n=1}^{N} \frac{1}{C_{o n}} .
$$

Substituting (1), (2), and (15) into (13) yields

$\left\{\begin{array}{l}\sum_{n=1}^{N}\left(\frac{L_{e n}}{\left(\omega_{t i}^{-}\right)^{-2}-\left(\omega_{p n}^{-}\right)^{-2}}-\frac{L_{o n}}{\left[\left(\omega_{t i}^{-}\right)^{-2}-\left(\omega_{p n}^{+}\right)^{-2}\right]\left(\frac{\omega_{t i}^{-}}{\omega_{p n}^{+}}\right)^{2}}\right)=-\frac{1}{C_{s h}} \\ \sum_{n=1}^{N}\left(\frac{L_{e n}}{\left(\omega_{t i}^{+}\right)^{-2}-\left(\omega_{p n}^{-}\right)^{-2}}-\frac{L_{o n}}{\left[\left(\omega_{t i}^{+}\right)^{-2}-\left(\omega_{p n}^{-}\right)^{-2}\right]\left(\frac{\omega_{t i}^{+}}{\omega_{p n}^{+}}\right)^{2}}\right)=-\frac{1}{C_{s h}}\end{array}\right.$

for $i=1,2, \ldots, N$. Equation (16) is a system of $2 N$ linear equations in the $2 N$ unknowns, $L_{e n}$ and $L_{o n}, n=1,2, \ldots, N$. In this equation, $\omega_{t i}^{-}$and $\omega_{t i}^{+}, i=1,2, \ldots, N$, are the $2 N$ specified transmission-zero frequencies; $\omega_{p n}^{-}$and $\omega_{p n}^{+}$, $n=1,2, \ldots, N$, are the $2 N$ resonant pole frequencies that are set iteratively, and $C_{s h}$ is the shunt capacitance in the core ICR network of the multiband BPF with a final value determined by a consistency condition.

Once the initial values of the parameters $\omega_{p n}^{-}, \omega_{p n}^{+}, n=$ $1,2, \ldots, N$, and $C_{s h}$ are given, (16) can be solved to obtain all of the element values of the even-mode and odd-mode half circuits in Fig. 4(a) and (b), except for the input capacitances $C_{e 0}$ and $C_{o 0}$. Since $C_{e 0}$ equals $C_{i n}$, as determined by comparing the two versions of the even-mode half circuits in Figs. 3(a) and 4(a), $C_{o 0}$ can be found by substituting (6) into (15) and eliminating $C_{i n}$ to yield

$$
C_{o 0}=\sum_{n=2}^{N} C_{f n}\left(1+\sqrt{1+\frac{2}{\sum_{n=2}^{N} C_{f n}\left(\sum_{n=1}^{N} \frac{1}{C_{o n}}-\frac{1}{C_{s h}}\right)}}\right)
$$


Upon closer inspection, (17) needs to be solved numerically because the capacitances $C_{f n}, n=2,3, \ldots, N$, that appear on the right hand side of this equation are derived from the residues of the odd-mode admittance, as described in (4), and therefore depend on $C_{o 0}$. In this work, Newton's method is applied to (17) to estimate $C_{o 0}$, and subsequently to calculate $C_{e 0}=C_{i n}$ using (6).

To satisfy the requirements of the specified reflection-zero frequency conditions, the values of $\omega_{p n}^{-}$and $\omega_{p n}^{+}$, $n=1,2, \ldots, N$, need to be iteratively updated until the nonlinear (14) is satisfied. Assume that the reflection zero frequencies $\omega_{r i}^{-}$and $\omega_{r i}^{+}$depend only on the resonant pole frequencies $\omega_{p n}^{-}$and $\omega_{p n}^{+}$, respectively, for $i=n$, and are independent of the others with $i \neq n$. The resonant pole resonant frequencies that are updated to satisfy (14) are derived in relation to their original frequencies as follows:

$$
\left\{\begin{array}{l}
\frac{j \omega_{r i}^{-} L_{e i}}{1-\left(\frac{\omega_{r i}^{-}}{\omega_{p i}^{-}}\right)^{2}}=\frac{j \omega_{r i}^{-} L_{e i}}{1-\left(\frac{\omega_{r i}^{-}}{\omega_{p i}^{-}}\right)^{2}}+\frac{Z_{0}^{2}-Z_{\text {even }}\left(\omega_{r i}^{-}\right) Z_{\text {odd }}\left(\omega_{r i}^{-}\right)}{Z_{\text {odd }}\left(\omega_{r i}^{-}\right)} \\
\frac{j \omega_{r i}^{+} L_{o i}}{1-\left(\frac{\omega_{r i}^{+}}{\omega_{p i}^{+\prime}}\right)^{2}}=\frac{j \omega_{r i}^{+} L_{o i}}{1-\left(\frac{\omega_{r i}^{+}}{\omega_{p i}^{+}}\right)^{2}}+\frac{Z_{0}^{2}-Z_{\text {even }}\left(\omega_{r i}^{+}\right) Z_{\text {odd }}\left(\omega_{r i}^{+}\right)}{Z_{\text {even }}\left(\omega_{r i}^{-}\right)}
\end{array}\right.
$$

for $i=1,2, \ldots, N$. In (18), $\omega_{p i}^{-\prime}$ and $\omega_{p i}^{+\prime}, i=1,2, \ldots, N$, denote the updated resonant pole frequencies. In fact, $\omega_{r i}^{-}$and $\omega_{r i}^{+}$depend most strongly on $\omega_{p i}^{-}$and $\omega_{p i}^{+}$, respectively, but also less strongly on $\omega_{p n}^{-}$and $\omega_{p n}^{+}$for $n \neq i$. Hence, (18) is iterated using the solutions of (16) and (17) until convergence to yield final estimates of the resonant pole frequencies. To ensure the convergence, the updated and original resonant pole frequencies are appropriately weighted to yield the new resonant pole frequencies for use in the iteration

$$
\left\{\begin{array}{l}
\omega_{p i}^{-\prime \prime}=w \omega_{p i}^{-}+(1-w) \omega_{p i}^{-\prime} \\
\omega_{p i}^{+\prime \prime}=w \omega_{p i}^{+}+(1-w) \omega_{p i}^{+\prime}, \quad i=1,2, \ldots, N
\end{array}\right.
$$

where $w$ is a weight parameter within the range of zero to one.

Finally, the value of $C_{s h}$ that is used in (16) must be reexamined for consistency. Since this value is an initial set value of the capacitance $C_{s h}$ in the odd-mode half circuit, it should be consistent with the value of the capacitance $C_{e 1}=C_{s h}$ in the even-mode half circuit. Therefore, in this work, a simple scan method is utilized to find the value of $C_{s h}$ that equals the one in $C_{e 1}$.

Alternatively, a capacitively coupled resonator (CCR) network can also be used as the core network of the proposed multiband BPF topology. However, if a CCR network-based topology is chosen, the shunt inductance $L_{s h}$ in the CCR network becomes the key element to assure the consistency condition in the synthesis procedure. Since an inductor usually occupies a large area and has significant parasitics in LTCC, the CCR network is therefore not preferred as compared to its counterpart ICR network.

Fig. 8 provides a flow chart of the procedure, discussed above, for designing the multiband BPF prototypes. It is emphasized that the first three steps in this flow chart are crucial to the success of this synthesis procedure. The first step of assigning

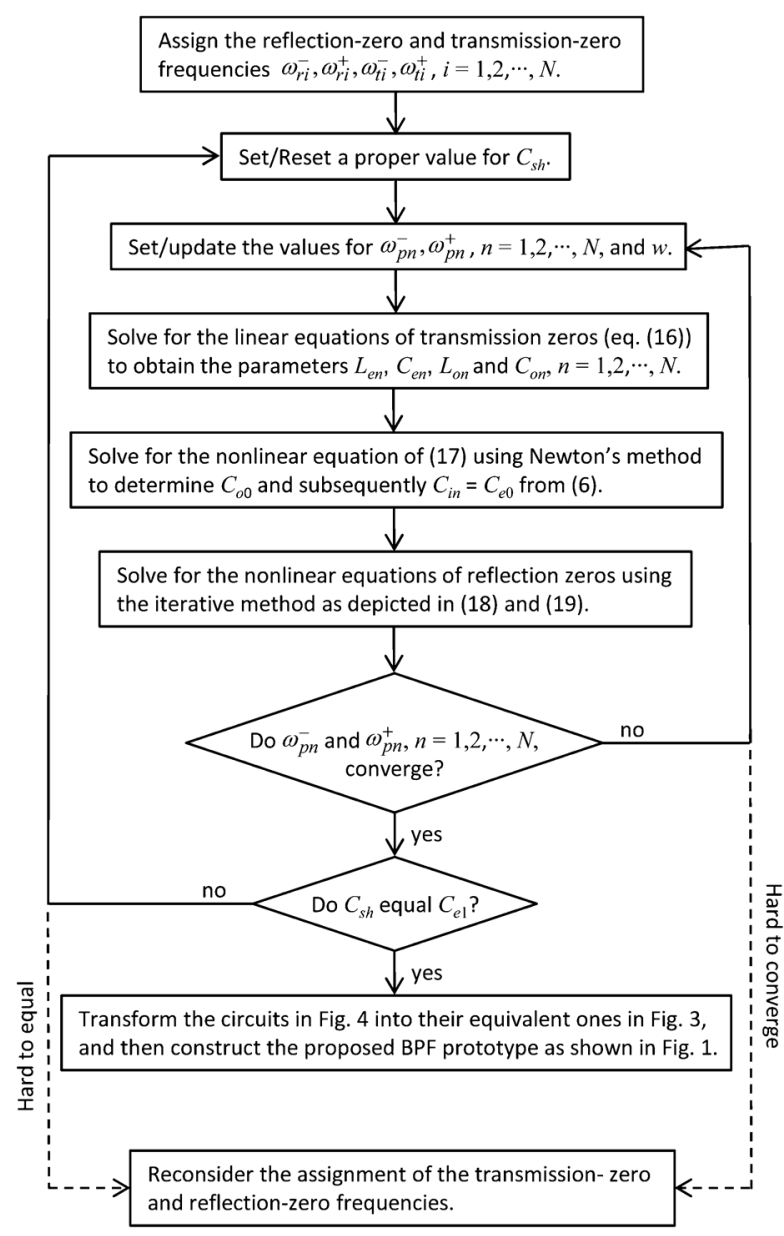

Fig. 8. Flow chart of the synthesis procedure for the multiband BPF prototype.

the transmission- and reflection-zero frequencies to the multiband BPF must be taken carefully because the proposed filter topology does not guarantee a solution for any of those frequencies, particularly when the transmission-zero frequencies are set too close to the reflection-zero frequencies. After all, the filter topology that is used herein is second-order, so relying on the transmission zeros to ensure high selectivity may not be practical. Nevertheless, the synthesizable transmission-zero frequencies provide the obvious advantage of suppressing the interference or spurious signals at multiple specific frequencies. Interestingly, moving adjacent passbands very close together to achieve a single higher-order passband is viable via a proper frequency assignment. As an illustrative demonstration, Fig. 9 shows the merging of the two adjacent second-order passbands of the penta-band BPF response in Fig. 7 into a single fourthorder passband in a quad-band BPF profile. However, the total number of usable transmission- and reflection-zero frequencies reduces. Then the system becomes under-determined and may have no solution or an infinite number of solutions.

In the second and third steps, the settings of the initial values of the variable parameters $C_{s h}, \omega_{p n}^{-}, \omega_{p n}^{+}$and $w$ are critical to the convergence of the iterative scheme used in this procedure. The initial value of $C_{s h}$ can use the design value from an ICR-based single-band LTCC BPF, as investigated in [26]. Additionally, setting $\omega_{r i}^{-}$and $\omega_{r i}^{+}$to the initial values of $\omega_{p n}^{-}$and $\omega_{p n}^{+}$, for 


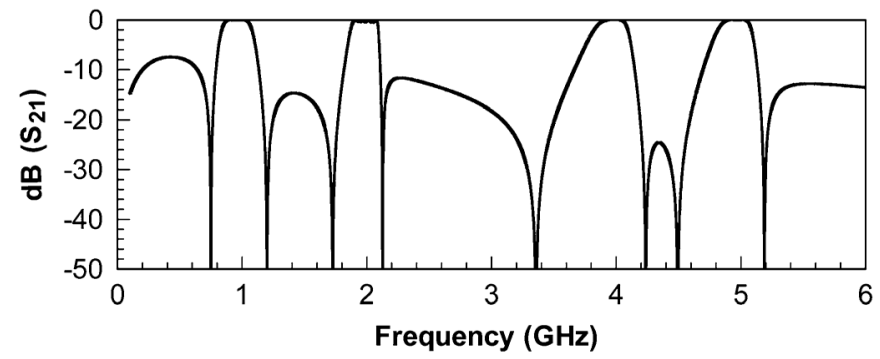

(a)

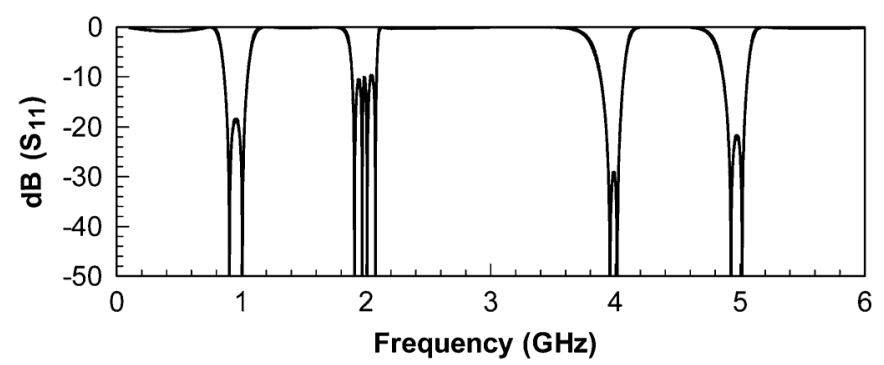

(b)

Fig. 9. Merging two adjacent second-order passbands of the penta-band BPF response in Fig. 7 into a single fourth-order passband in a quad-band BPF profile. Note that the used parameters are the same as those for Fig. 7 except the following ones: $\left(\omega_{p 2}^{-}, \omega_{p 3}^{-}\right)=2 \pi \times(1.9 \mathrm{GHz}, 2.03 \mathrm{GHz}) ;\left(\omega_{p 2}^{+}, \omega_{p 3}^{+}\right)=$ $2 \pi \times(1.975 \mathrm{GHz}, 2.1 \mathrm{GHz}) ; L_{e n}=5 / 24 \mathrm{nH}$ and $L_{o n}=1 / 12 \mathrm{nH}$, for $n=2$ and 3. (a) Magnitude of $S_{21}$. (b) Magnitude of $S_{11}$.

$i=n$, is a good idea. Furthermore, a larger weight parameter $w$ corresponds to faster convergence, but also greater probability of divergence. In the implementation of this synthesis procedure, $w$ is set to 0.5 , based on empirical results.

\section{DESIGN IMPLEMENTATION}

To validate the procedure that was described in Section III for synthesizing a multiband BPF prototype, which is shown in Fig. 1, a dual-band design and a tri-band design were implemented using this procedure. The synthesized prototypes were structured in LTCC with fully three-dimensional (3-D) lumped elements. This section provides details of the implementation of both designs and the structures thus obtained.

\section{A. Dual-Band Design}

For 2.4 and 5.2 GHz Wireless Local Area Network (WLAN) dual-band applications, the required passbands of the dual-band BPF must cover frequencies from 2.4 to $2.5 \mathrm{GHz}$ and frequencies from 5.15 to $5.35 \mathrm{GHz}$. However, given the possibility of a shift in the central passband frequencies owing to fabrication tolerance, a larger passband bandwidth than required is set by setting $\omega_{r \cdot 1}=2 \pi \times 2.3 \mathrm{GHz}, \omega_{r 2}=2 \pi \times 2.6 \mathrm{GHz}$, $\omega_{r 3}=2 \pi \times 5.1 \mathrm{GHz}$, and $\omega_{r 4}=2 \pi \times 5.4 \mathrm{GHz}$. Moreover, considering the suppression of the interference signals from the Integrated Services Digital Broadcasting-Terrestrial (ISDB-T) and the Digital Video Broadcasting-Handheld (DVB-H) band of $0.47-0.87 \mathrm{GHz}$ and the Worldwide Interoperability for Microwave Access (WIMAX) band of 3.3-3.8 GHz, the desired stopband performance is achieved by setting $\omega_{t 1}=2 \pi \times 1 \mathrm{GHz}$, $\omega_{t 2}=2 \pi \times 2.9 \mathrm{GHz}, \omega_{t 3}=2 \pi \times 4 \mathrm{GHz}$, and $\omega_{t 4}=2 \pi \times$ 6.3 GHz.

In addition to the setting of the above four reflection-zero frequencies and four transmission-zero frequencies, the value of

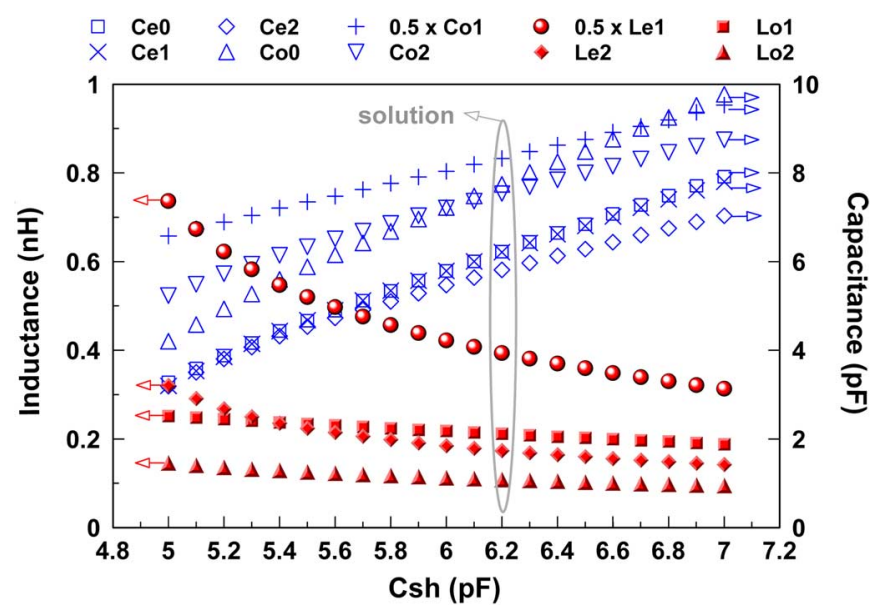

Fig. 10. Convergent element values of the even-mode and odd-mode half circuits for the dual-band BPF prototype with different set values of $C_{s h}$.

$C_{s h}$ is set within a range from 5 to $7 \mathrm{pF}$ in steps of $0.1 \mathrm{pF}$, and the weight parameter $w$ is a fixed value of 0.5 . Furthermore, the four designated reflection-zero frequencies $2 \pi \times 2.3 \mathrm{GHz}$, $2 \pi \times 2.6 \mathrm{GHz}, 2 \pi \times 5.1 \mathrm{GHz}$, and $2 \pi \times 5.4 \mathrm{GHz}$ are used as the initial values of $\omega_{p 1}^{-}, \omega_{p 1}^{+}, \omega_{p 2}^{-}$and $\omega_{p 2}^{+}$, respectively. When these data are input to the synthesis procedure, which is presented in Fig. 8, all of the values of the elements of the even-mode and odd-mode half circuits in Fig. 4(a) and (b) are as displayed in Fig. 10, as $\omega_{p 1}^{-}, \omega_{p 1}^{+}, \omega_{p 2}^{-}$and $\omega_{p 2}^{+}$all converge to stable values. In this figure, the circled data points represent the obtained element values that satisfy the consistency condition $C_{s h}=C_{e 1}=6.2 \mathrm{pF}$.

Fig. 11 shows the convergence of the resonant pole frequencies at $C_{s h}=6.2 \mathrm{pF}$; it demonstrates that the resonant pole frequencies starts as the reflection-zero frequencies and are iteratively adjusted until these reflection-zero frequencies satisfy the condition in (14). The iterative process takes about 12 steps to converge. Fig. 12 plots the $S$-parameter magnitude responses of the prototype solution found in Fig. 10, further verifying that in these responses, the transmission and reflection zeros are at the specified frequencies.

\section{B. Tri-Band Design}

The tri-band BPF design to be presented here has an additional third band for $800 \mathrm{MHz}$ long term evolution (LTE) applications because it has one more pair of $L_{g 3} C_{g 3}$ and $L_{f 3} C_{f 3}$ resonators than the dual-band BPF design has. To yield three passbands that cover the necessary frequency ranges of $2.4-2.5 \mathrm{GHz}, 5.15-5.35 \mathrm{GHz}$, and 790-882 MHz, the reflection zeros for this tri-band BPF design are at frequencies of $\omega_{r 1}=2 \pi \times 2.35 \mathrm{GHz}, \omega_{r 2}=2 \pi \times 2.55 \mathrm{GHz}, \omega_{r 3}=$ $2 \pi \times 5.1 \mathrm{GHz}, \omega_{r 4}=2 \pi \times 5.4 \mathrm{GHz}, \omega_{r 5}=2 \pi \times 0.74 \mathrm{GHz}$, and $\omega_{r 6}=2 \pi \times 0.94 \mathrm{GHz}$. To desire that the three passbands exhibit a symmetric rolloff response, the transmission zeros are symmetrically located on the two sides of each passband with spacings of $0.9,1.3$, and $0.6 \mathrm{GHz}$ for the first to the third bands, respectively. Therefore, the transmission-zero frequencies are set as $\omega_{t 1}=2 \pi \times 2 \mathrm{GHz}, \omega_{t 2}=2 \pi \times 2.9 \mathrm{GHz}$, $\omega_{t 3}=2 \pi \times 4.6 \mathrm{GHz}, \omega_{t 4}=2 \pi \times 5.9 \mathrm{GHz}, \omega_{t 5}=2 \pi \times 0.5 \mathrm{GHz}$, and $\omega_{t 6}=2 \pi \times 1.1 \mathrm{GHz}$. 


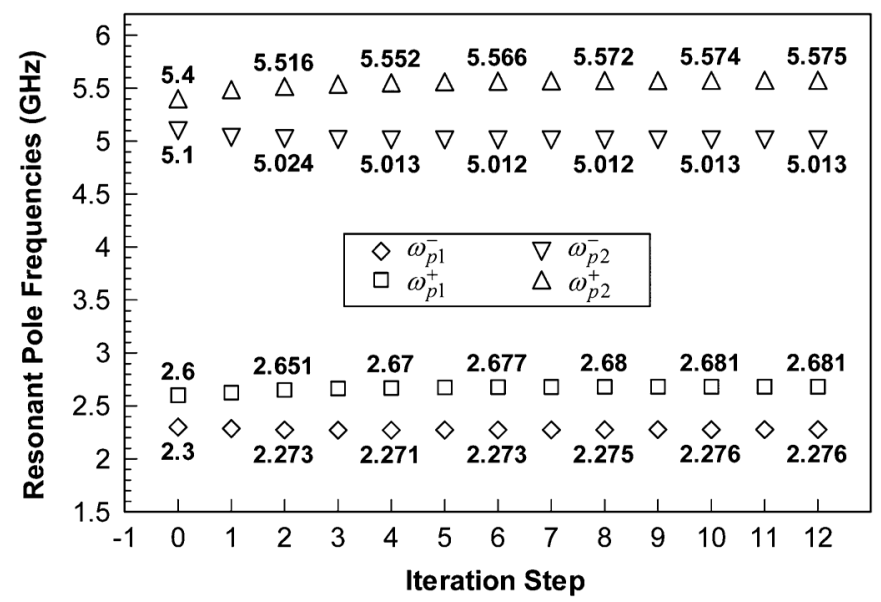

Fig. 11. Convergence of the resonant pole frequencies of the even-mode and odd-mode half circuits in the iterative approach to the solution of the dual-band BPF prototype with $C_{s h}=6.2 \mathrm{pF}$

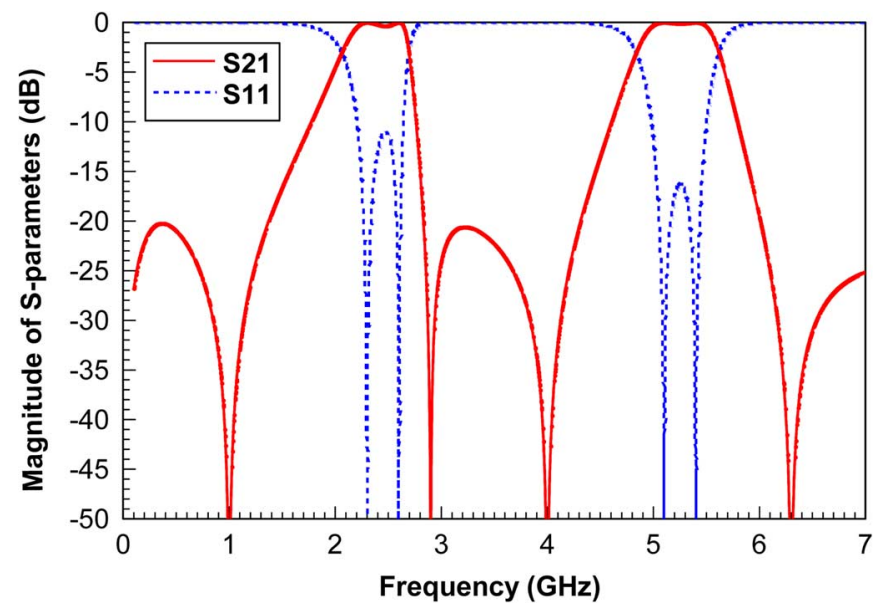

Fig. 12. $S$-parameter magnitude responses of the obtained dual-band BPF prototype solution.

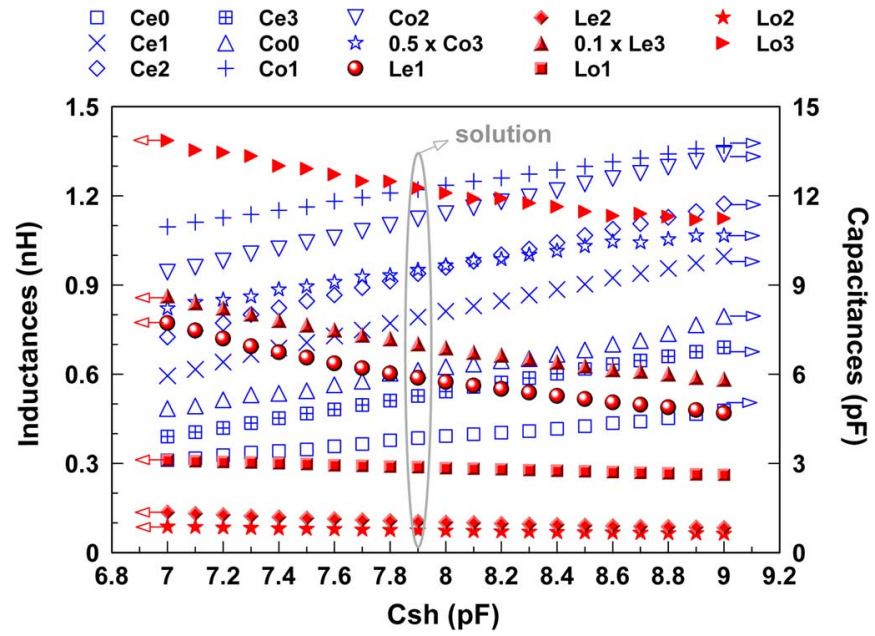

Fig. 13. Convergent element values of the even-mode and odd-mode half circuits for the tri-band BPF prototype with different set values of $C_{s h}$.

In this tri-band design, the values of $C_{s h}$ range from 7 to $9 \mathrm{pF}$ in steps of $0.1 \mathrm{pF}$, while the value of $w$ is kept at 0.5 . The initial values of the resonant pole frequencies are also set to the reflection-zero frequencies. Consequently, Fig. 13 shows the converged values of elements in the even-mode and odd-mode half

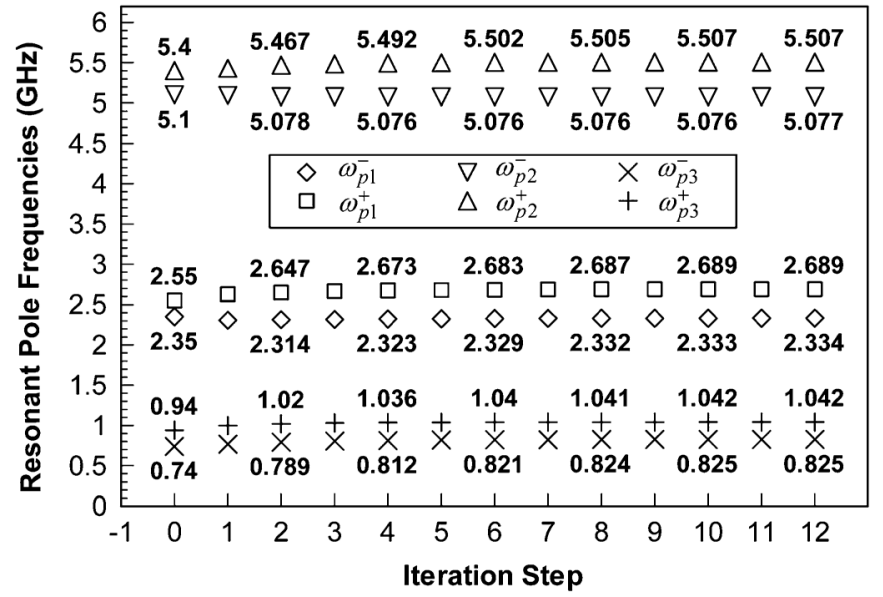

Fig. 14. Convergence of the resonant pole frequencies of the even-mode and odd-mode half circuits in the iterative approach to the solution of the tri-band BPF prototype with $C_{s h}=7.9 \mathrm{pF}$.

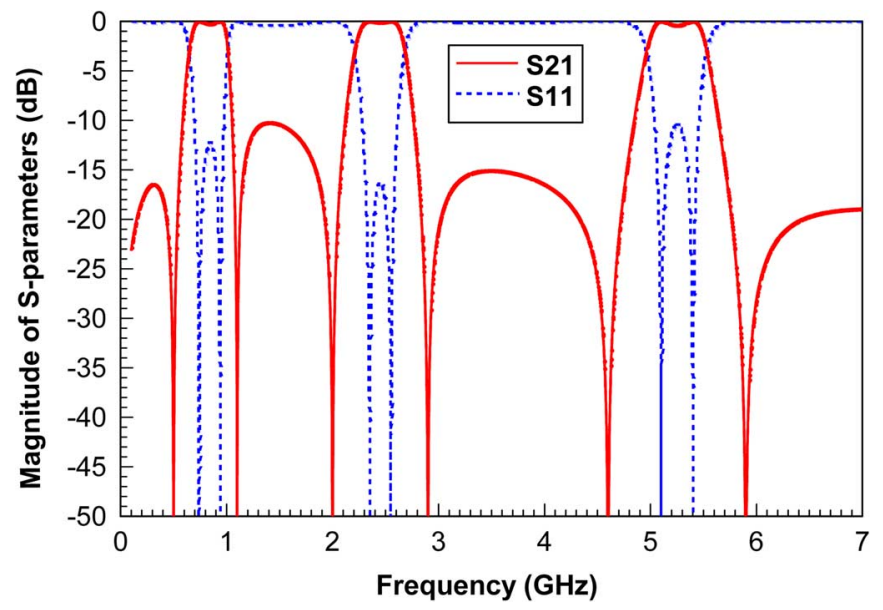

Fig. 15. $S$-parameter magnitude responses of the obtained tri-band BPF prototype solution.

circuits. Similarly, the data points that are circled in this figure represent the desired element values with equivalent $C_{s h}=$ $C_{e 1}=7.9 \mathrm{pF}$. Fig. 14 reveals how the resonant pole frequencies change in each iteration for $C_{s h}=7.9 \mathrm{pF}$. All of the resonant pole frequencies converge in approximately a dozen iterations. Fig. 15 displays the calculated magnitudes of $S$-parameters according to the prototype solution found in Fig. 13. These responses confirm that the frequencies of the transmission and reflection zeros are those specified in the design.

\section{LTCC Structure}

In this work, the designed BPFs were structured using the Heraus LTCC process with a dielectric constant of 7.8, a loss tangent of 0.003 , and a layer thickness of $27 \mu \mathrm{m}$. The conductors are silver with a thickness of $10 \mu \mathrm{m}$. In the implementation of the synthesized BPF prototypes using lumped elements, some of the elements are split into two equivalent ones in series or in parallel to maintain symmetry. Table I lists the element values required by the synthesized dual-band and tri-band BPFs and the descriptions of the physical structures of the elements. Basically, all of the elements are either inductors or capacitors in a one-to-three layer structure. Most of the inductors have a 
TABLE I

VAlues AND Structures of the Circuit Elements of THE DUAL-BAND AND TRI-BAND BPFS

\begin{tabular}{|c|c|c|}
\hline \multicolumn{2}{|c|}{ Dual-band BPF prototype } & \multirow{2}{*}{ Element physical structure } \\
\hline Element & Value & \\
\hline$L_{s e} / 2$ & $1.02 \mathrm{nH}$ & Spiral \\
\hline$C_{\text {in }}$ & $6.22 \mathrm{pF}$ & Parallel plate \\
\hline$L_{s h}$ & $787 \mathrm{pH}$ & Spiral \\
\hline$C_{\text {sh }}$ & $6.2 \mathrm{pF}$ & 3 stacked plate interdigital \\
\hline$L_{g 2}$ & $86.7 \mathrm{pH}$ & Via \\
\hline$C_{g 2} / 2$ & $5.81 \mathrm{pF}$ & Parallel plate \\
\hline$L_{f 2} / 2$ & $854 \mathrm{pH}$ & Spiral \\
\hline $2 C_{f 2}$ & $1.52 \mathrm{pF}$ & Parallel plate \\
\hline \multicolumn{2}{|c|}{ Tri-band BPF prototype } & \multirow{2}{*}{ Element physical structure } \\
\hline Element & Value & \\
\hline$L_{s e} / 2$ & $1.4 \mathrm{nH}$ & Spiral \\
\hline$C_{i n}$ & $3.84 \mathrm{pF}$ & Parallel plate \\
\hline$L_{s h}$ & $587 \mathrm{pH}$ & Spiral \\
\hline$C_{s h}$ & $7.9 \mathrm{pF}$ & 3 stacked plate interdigital \\
\hline$L_{g 2}$ & $52.4 \mathrm{pH}$ & Via \\
\hline$C_{g 2} / 2$ & $9.38 \mathrm{pF}$ & 3 stacked plate interdigital \\
\hline $2 L_{g 3}$ & $7.03 \mathrm{nH}$ & 2 stacked Spiral \\
\hline$C_{g 3} / 2$ & $5.27 \mathrm{pF}$ & Parallel plate \\
\hline$L_{f 2} / 2$ & $1.75 \mathrm{nH}$ & Spiral \\
\hline $2 C_{f 2}$ & $605 \mathrm{fF}$ & Parallel plate \\
\hline$L_{f 3} / 2$ & $18.7 \mathrm{nH}$ & $\begin{array}{c}L_{f 3}^{\prime} / 2 \text { and } 2 C_{L f 3} \text { in parallel } \\
L_{f 3} / 2: 3 \text { stacked spiral } \\
2 C_{L f 3}: \text { parallel plate }\end{array}$ \\
\hline $2 C_{f 3}$ & $1.68 \mathrm{pF}$ & Parallel plate \\
\hline
\end{tabular}

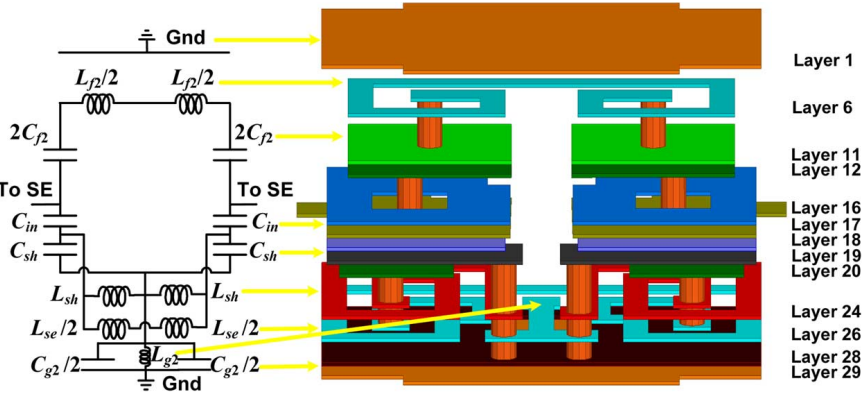

Fig. 16. Schematic of lumped-element implementation and 3-D LTCC structure of the designed dual-band BPF. Note that SE stands for side electrode.

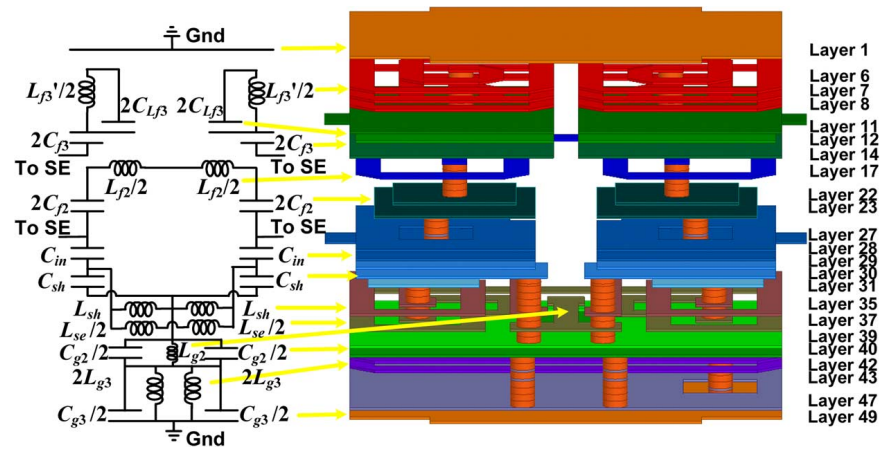

Fig. 17. Schematic of lumped-element implementation and 3-D LTCC structure of the designed tri-band BPF.

spiral shape in a single or stacked configuration. A few inductors have a via conductor structure because they have very small

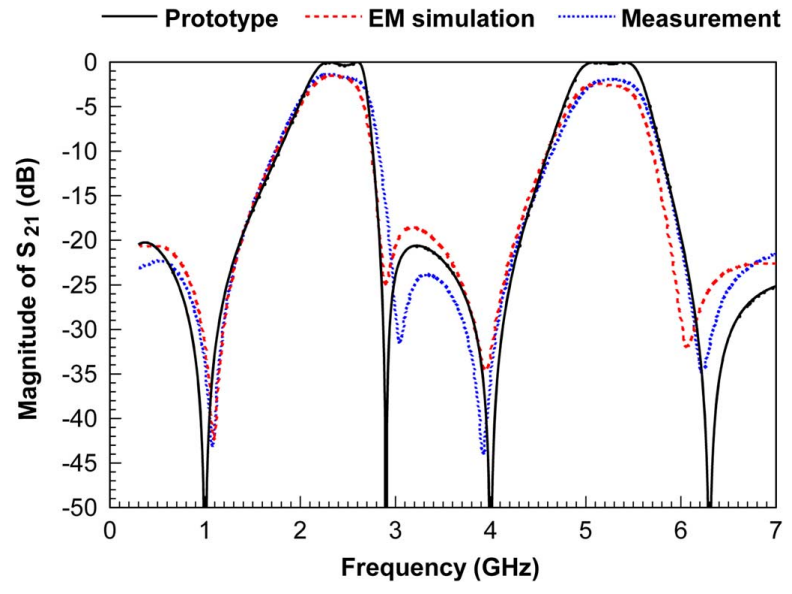

Fig. 18. Comparison of the magnitudes of $S_{21}$-parameter among the synthesized prototype, EM simulation and measurement for the designed dual-band LTCC BPF.

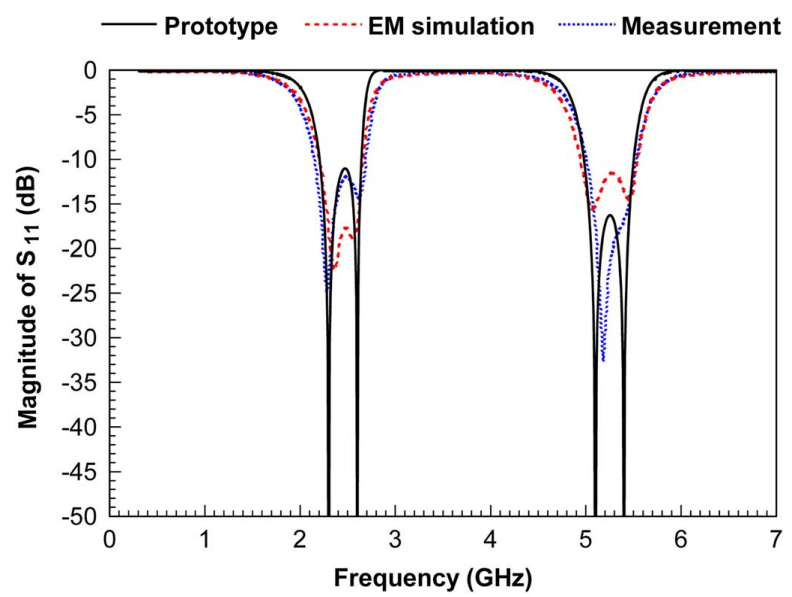

Fig. 19. Comparison of the magnitudes of $S_{11}$-parameter among the synthesized prototype, EM simulation and measurement for the designed dual-band LTCC BPF.

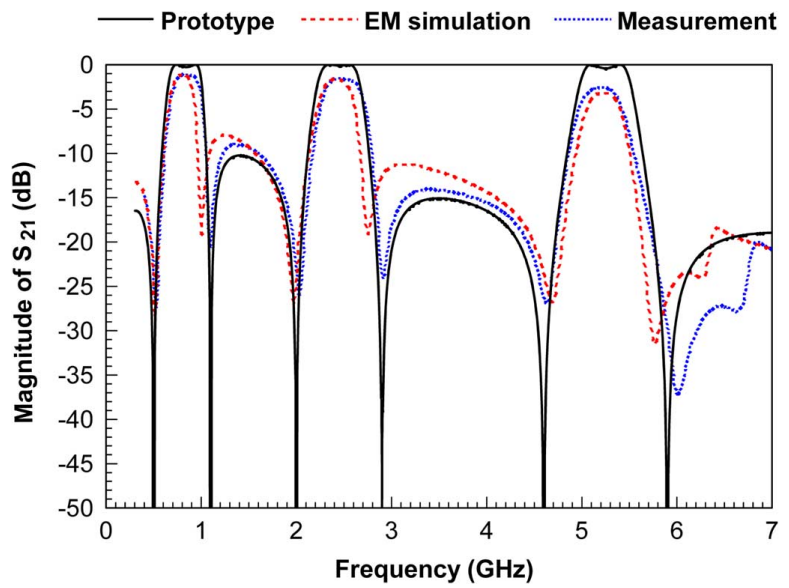

Fig. 20. Comparison of the magnitudes of $S_{21}$-parameter among the synthesized prototype, EM simulation and measurement for the designed tri-band LTCC BPF.

inductance values. The capacitors form a parallel-plate or a vertically-stacked-plate interdigital structure. Notably, these physical configurations of inductors and capacitors did not yield exactly the element values that are provided in Table I. A change in most of the element values of less than ten percent compensates for the self-parasitics of the individual elements and the 
TABLE II

Performance Comparison of this Work With Previous Multiband LTCC BPF Designs

\begin{tabular}{|c|c|c|c|c|c|c|c|c|}
\hline \multirow[b]{2}{*}{ Ref. } & \multirow{2}{*}{ Type } & \multicolumn{4}{|c|}{ Passband Specifications } & \multirow{2}{*}{$\begin{array}{c}\text { Transmission-zero } \\
\text { Frequencies }\end{array}$} & \multirow[b]{2}{*}{ Size } & \multirow{2}{*}{ Filter Structure } \\
\hline & & Center freq. & $\mathrm{FBW}^{\&}$ & Insertion loss & Return loss & & & \\
\hline \multirow[b]{2}{*}{ [6] } & \multirow{2}{*}{$\begin{array}{l}\text { Dual-band } \\
\text { BPF }\end{array}$} & $2.2 \mathrm{GHz}$ & $12.1 \% *$ & $1.8 \mathrm{~dB}$ at $2.2 \mathrm{GHz}$ & $>10 \mathrm{~dB}$ within FBW & \multirow{2}{*}{$3.17 \mathrm{GHz}$} & \multirow{2}{*}{$\begin{array}{c}2.5 \times 2.0 \times 0.94 \\
\mathrm{~mm}^{3}\end{array}$} & \multirow{2}{*}{$\begin{array}{l}\text { Combined structure of } \\
\text { two single-band BPF }\end{array}$} \\
\hline & & $5.4 \mathrm{GHz}$ & $27.5 \% *$ & $1 \mathrm{~dB}$ in $4.9-5.9 \mathrm{GHz}$ & $>10 \mathrm{~dB}$ within $\mathrm{FBW}$ & & & \\
\hline \multirow[b]{2}{*}[7]{} & \multirow{2}{*}{$\begin{array}{l}\text { Dual-band } \\
\text { BPF }\end{array}$} & $3.96 \mathrm{GHz}$ & $40 \%$ & $<2.5 \mathrm{~dB}$ within $\mathrm{FBW}$ & $>4.7 \mathrm{~dB}$ within $\mathrm{FBW}^{*}$ & \multirow{2}{*}{$\begin{array}{c}1.72,5.03,5.37 \text { and } \\
12.13 \mathrm{GHz}^{*}\end{array}$} & \multirow{2}{*}{$\begin{array}{c}7.5 \times 3.8 \times 0.386 \\
\mathrm{~mm}^{3}\end{array}$} & \multirow{2}{*}{$\begin{array}{l}\text { Combined structure of } \\
\text { two single-band BPF }\end{array}$} \\
\hline & & $7.92 \mathrm{GHz}$ & $40 \%$ & $<2.5 \mathrm{~dB}$ within $\mathrm{FBW}$ & $>6.1 \mathrm{~dB}$ within $\mathrm{FBW}^{*}$ & & & \\
\hline \multirow[b]{2}{*}[8]{} & \multirow{2}{*}{$\begin{array}{c}\text { Dual-band } \\
\text { BPF }\end{array}$} & $2.54 \mathrm{GHz}$ & $8.1 \%$ & $<3.7 \mathrm{~dB}$ within $\mathrm{FBW}$ & $>7.6 \mathrm{~dB}$ within $\mathrm{FBW}^{*}$ & \multirow{2}{*}{$\begin{array}{c}2.99 \text { and } 6.23 \\
\mathrm{GHz}^{*}\end{array}$} & \multirow{2}{*}{$\begin{array}{c}2.69 \times 1.78 \\
\mathrm{~mm}^{2}\end{array}$} & \multirow{2}{*}{$\begin{array}{l}\text { Coupled semi-lumped } \\
\text { resonator structure }\end{array}$} \\
\hline & & $5.38 \mathrm{GHz}$ & $5.7 \%$ & $<4.45 \mathrm{~dB}$ within $\mathrm{FBW}$ & $>8.7 \mathrm{~dB} \mathrm{w}$ & & & \\
\hline \multirow{2}{*}{ [9] } & \multirow{2}{*}{$\begin{array}{c}\text { Dual-band } \\
\text { BPF }\end{array}$} & $2.59 \mathrm{GHz}$ & $26 \%$ & $<2.1 \mathrm{~dB}$ within $\mathrm{FBW}$ & $>12 \mathrm{~dB} \mathrm{w}$ & \multirow{2}{*}{$\begin{array}{l}0.51,3.28,3.62, \\
4.2 \text { and } 9 \mathrm{GHz}^{*}\end{array}$} & \multirow{2}{*}{$\begin{array}{c}5.1 \times 4.6 \times 0.65 \\
\mathrm{~mm}^{3}\end{array}$} & \multirow{2}{*}{$\begin{array}{l}\text { Coupled semi-lumped } \\
\text { resonator structure }\end{array}$} \\
\hline & & $5.09 \mathrm{GHz}$ & $15 \%$ & in $\mathrm{FBW}$ & $>12 \mathrm{~dB} \mathrm{v}$ & & & \\
\hline \multirow{2}{*}[10]{} & \multirow{2}{*}{$\begin{array}{c}\text { Dual-band } \\
\text { BPF }\end{array}$} & $2.4 \mathrm{GHz}$ & $16.7 \%$ & $<1.25 \mathrm{~dB} v$ & $>24.6 \mathrm{~dB}$ & \multirow{2}{*}{ None } & \multirow{2}{*}{$\begin{array}{c}15 \times 13 \\
\mathrm{~mm}^{2}\end{array}$} & \multirow{2}{*}{$\begin{array}{c}\text { Coupled SIR resonator } \\
\text { structure }\end{array}$} \\
\hline & & $5.18 \mathrm{GHz}$ & $12.3 \%$ & $<1.87 \mathrm{dl}$ & $>11.2 \mathrm{~dB}$ & & & \\
\hline \multirow{2}{*}{ [11] } & Dual-band & $2.33 \mathrm{GHz}$ & $52 \%$ & $<1.3 \mathrm{dl}$ & $>15 \mathrm{~dB}$ & & $11.1 \times 14.4 \times 0.58$ & Coupled SIR resonator \\
\hline & & $5.76 \mathrm{GHz}$ & $18 \%$ & in FBW & $>15 \mathrm{~dB}$ & & & \\
\hline & Dual-band & $29.47 \mathrm{GHz}$ & $4.5 \%$ & $<4.5 \mathrm{~dB} v$ & $>11.6 \mathrm{~dB} \mathrm{w}$ & d 35.66 & $5 \times 4 \times 0.652$ & vity \\
\hline [13] & $\mathrm{BPF} \# 1$ & $34.54 \mathrm{GHz}$ & $4.1 \%$ & n FBW & $>10 \mathrm{~dB}$ wi & & & \\
\hline$[13]$ & Dual-band & $30.3 \mathrm{GHz}$ & $6 \%$ & $<4.8 \mathrm{~dB}$ within FBW & $>12 \mathrm{~dB}$ within $\mathrm{FBW} *$ & $28.4,31.56,37.17$ & $4.12 \times 3.92 \times 0.832$ & vity \\
\hline & & $39.3 \mathrm{GHz}$ & $6 \%$ & hin FBW & $>11.3 \mathrm{~dB}$ within $\mathrm{FBW}^{*}$ & & & \\
\hline & & $30 \mathrm{GHz}$ & $4 \%$ & $4.09 \mathrm{~dB}$ at $30 \mathrm{GHz}$ & $>12.5 \mathrm{~dB}$ & & & \\
\hline [14] & & $35 \mathrm{GHz}$ & $2.8 \%$ & $5.18 \mathrm{~dB}$ at $35 \mathrm{GHz}$ & $>5.6 \mathrm{~dB} \mathrm{w}$ & $32.21 \mathrm{GHz}^{*}$ & 2 & \\
\hline & & 37 & $2.8 \%$ & 6.44 & $>5$ & & & \\
\hline & ual-band & $2.45 \mathrm{GHz}$ & $12.2 \%{ }^{\#}$ & FBW & $>12 \mathrm{~dB}$ & $.07,3.04,3.92$ an & $2 \times 1.25 \times 0.9$ & able \\
\hline & & 5.25 & $5.7 \%^{\#}$ & $<2.3 \mathrm{~d}$ & $>16.8$ & & & \\
\hline & & $2.45 \mathrm{GHz}$ & $8.2 \%^{\#}$ & $<2 \mathrm{~dB}$ within $\mathrm{FBW}$ & $>14.6 \mathrm{~dB}$ within FBW & & & \\
\hline & & $5.25 \mathrm{GHz}$ & $5.7 \%^{\#}$ & $<3.3 \mathrm{~dB}$ within $\mathrm{FBW}$ & $>11.3$ & $2.91,4.62$ and & 6 & ible \\
\hline & & $0.84 \mathrm{GHz}$ & $23.8 \%{ }^{\#}$ & $<1.6 \mathrm{~dB}$ within FBW & $>15.1 \mathrm{~d}$ & & & \\
\hline
\end{tabular}

\& defined as the bandwidth normalized by the center frequency

\# estimated from the spacings of the two designated reflection-zero frequencies for individual passbands

* graphically estimated

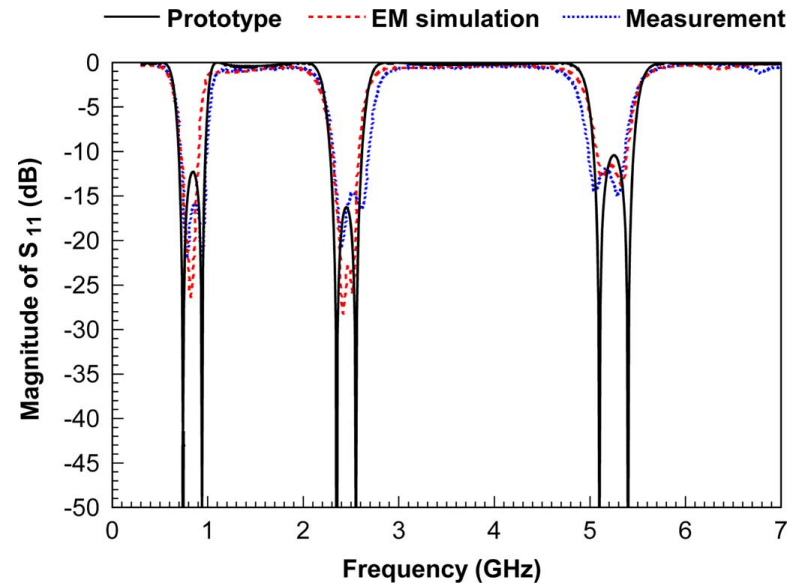

Fig. 21. Comparison of the magnitudes of $S_{11}$-parameter among the synthesized prototype, EM simulation and measurement for the designed tri-band LTCC BPF.

parasitics of interconnections. The exception is the inductance of inductor $L_{f 3} / 2$, which is exceptionally large and must be especially considered. This inductor is substituted by an inductor of $7.6 \mathrm{nH}$ in parallel with a capacitor of $1.82 \mathrm{pF}$ to maintain the same resonant pole frequency of $\omega_{p 3}^{+}=2 \pi \times 1.042 \mathrm{GHz}$, which is shown in Fig. 14 as a final estimate of $\omega_{p 3}^{+}$in the iterative process.

Figs. 16 and 17 show the schematics of lumped-element implementation and 3-D LTCC structures of the designed dual-band and tri-band BPFs, respectively. Clearly, in these two structures, the core ICR network elements are placed in the middle layers, while the elements that are used in the parallel $L_{g n} C_{g n}$ and series $L_{f n} C_{f n}$ resonators are placed in the lower and higher layers, respectively. All of the conductors are distributed through 13 out of 29 layers for the dual-band LTCC BPF and 23 out of 49 layers for the tri-band LTCC BPF. More layers than necessary are used in building the metallic structures of the BPFs to reduce the parasitic shunt capacitances of the elements to ground as well as to reduce the mutual coupling of the elements. This leads to accurate and robust frequency responses of the various resonators in the results of electromagnetic (EM) simulation with isolated excitation. Both dual-band and tri-band LTCC BPFs have the same area of $2 \times 1.25 \mathrm{~mm}^{2}$ because their core ICR networks are similar, but their thicknesses differ because they require different numbers of layers to accommodate the feedback resonant networks. The dual-band LTCC BPF is $0.9 \mathrm{~mm}$ thick, while the tri-band LTCC $\mathrm{BPF}$ is $1.6 \mathrm{~mm}$ thick.

\section{COMPARISON OF RESULTS AND DISCUSSION}

For the purpose of measurement, the fabricated LTCC BPFs were surface-mounted onto a printed circuit board. Then, with the help of the Thru-Reflect-Line (TRL) calibration process, the $S$-parameters were measured by the network analyzer. For the dual-band LTCC BPF, Figs. 18 and 19 compare the magnitudes of the $S_{11}$ and $S_{21}$ parameters that are predicted using the prototype, obtained by the EM simulations using Ansys HFSS, and measured. The comparison indicates that the prototype responses, except for the dissipative loss, are very close to the EM-simulated and measured responses. In addition, the four reflection zeros are measured at 2.28, 2.62, 5.18, and $5.38 \mathrm{GHz}$, and the four transmission zeros are measured at 1.07, 3.04, 3.92, and $6.21 \mathrm{GHz}$. The maximum deviations of the reflection and transmission zeros from their specified frequencies are 80 and 
$140 \mathrm{MHz}$, respectively. Based on the measured results, the key specifications of this dual-band LTCC BPF are summarized as follows. The insertion loss is less than $1.8 \mathrm{~dB}$ in the $2.4-2.5 \mathrm{GHz}$ band and less than $2.1 \mathrm{~dB}$ in the $5.15-5.35 \mathrm{GHz}$ band. The return loss exceeds $11 \mathrm{~dB}$ in both bands. The attenuation exceeds $20 \mathrm{~dB}$ in the stopband from 0.3 to $1.36 \mathrm{GHz}$, from 2.93 to $4.27 \mathrm{GHz}$, and above $6 \mathrm{GHz}$.

Figs. 20 and 21 present the $S$-parameters of the tri-band LTCC BPF. Again, close agreement can be obtained among the results from the prototype predictions, EM simulations and measurements if the dissipative loss is not considered. The measured results show that the six reflection-zero frequencies are $0.78,0.94,2.4,2.62,5.05$, and $5.29 \mathrm{GHz}$, and the six transmission-zero frequencies are $0.52,1.09,2.03,2.91,4.62$, and $6.02 \mathrm{GHz}$. The maximum deviations of the reflection-zero and transmission-zero frequencies from the designated frequencies are 110 and $120 \mathrm{MHz}$, respectively. The measured results also indicate that this tri-band LTCC BPF has the following important specifications. The insertion loss is smaller than 1.2, 1.6 and $2.8 \mathrm{~dB}$ within the $790-882 \mathrm{MHz}$ band, $2.4-2.5 \mathrm{GHz}$ band and $5.15-5.35 \mathrm{GHz}$ band, respectively. The return loss is greater than $12 \mathrm{~dB}$ in all three bands. Due to a setting of the transmission-zero frequencies closer to the passbands, the tri-band LTCC achieves a steeper rolloff from each passband to the stopband than does the dual-band LTCC BPF. However, this advantage is at the expense of an increased minimum attenuation between the two adjacent transmission-zero frequencies in the stopband. Fig. 20 shows that the tri-band LTCC BPF has local minima of attenuation with values of 9 and $14 \mathrm{~dB}$ at 1.36 and $3.42 \mathrm{GHz}$, respectively.

Table II compares the measured performance between this work and the previous works on multiband LTCC BPFs. The comparison reveals that the presented dual-band BPF has comparable insertion and return losses in the passbands and numbers of transmission zeros in the stopbands to the other dual-band ones, but the proposed design achieves the smallest area. Moreover, this work demonstrates a superior capability to extend the LTCC BPF design from the dual-band to the tri-band under the same area constraint.

\section{CONCLUSION}

This paper proposed a novel topology and a synthesis procedure for designing multiband LTCC BPFs. The filter topology uses an ICR circuit as the core network and extendible multistage resonant circuits as series- and parallel-feedback networks to configure a multiband BPF prototype with an expandable number of operating bands. The passbands and stopbands are highly configurable by varying the synthesizable reflection- and transmission-zero frequencies. The proposed synthesis procedure was utilized to establish a dual-band and a tri-band BPF prototypes for WLAN and LTE applications, which are implemented in LTCC with 3-D lumped elements. EM simulations and experimental results validate the presented methods and demonstrate the compact size of the designed LTCC BPFs.

\section{ACKNOWLEDGMENT}

The authors would like to thank the Chilisin Electronics Corporation, Hsinchu, Taiwan, for providing the fabrication service of the LTCC BPF designs.

\section{REFERENCES}

[1] Y. Andoh, "Market and technology of RF modules: Focusing on front end modules for cellular terminals," in Proc. CS Mantech Conf., 2010, pp. 21-24.

[2] R. Gómez-García, J.-M. Muñoz-Ferreras, and M. Sánchez-Renedo, "Microwave transversal six-band bandpass planar filter for multistandard wireless applications," in Proc. IEEE Radio Wireless Symp., 2011, pp. 166-169.

[3] K.-W. Hsu, J.-H. Lin, and W.-H. Tu, "Compact sext-band bandpass filter with sharp rejection response," IEEE Microw. Wireless Compon. Lett., vol. 24, no. 9, pp. 593-595, Sep. 2014.

[4] C.-H. Chen, C.-H. Huang, T.-S. Horng, and S.-M. Wu, "Highly miniaturized multiband bandpass filter design based on a stacked spiral resonator structure," IEEE Trans. Microw. Theory Techn., vol. 60, no. 5 , pp. 1278-1286, May 2012.

[5] C.-H. Chen and K.-H. Lin, "Novel 3-D space partition method for triple WiMAX multilayer LTCC diplexer IPD design with EM coupling sensitivity concerns," in Proc. IEEE Region 10 Conf., 2007, pp. $1-4$.

[6] D. Kim, J. I. Ryu, H.-M. Cho, N.-K. Kang, and J. C. Kim, "Implementation of a highly integrated triple-band RF front-end module," in Proc. Asia-Pac. Microw. Conf., 2007, pp. 265-268.

[7] T. W. Kim and Y. C. Lee, "A compact sized LTCC diplexer with high-band selectivity and high isolation for GSM and CDMA multiband applications," in Proc. Asia-Pac. Microw. Conf., 2009, pp. 2080-2083.

[8] B. C. Ham, D. H. Kim, J. M. Yook, J. I. Ryu, J. C. Kim, J. C. Park, Y. C. Park, and D. Kim, "A GPS/BT/WiFi triple-mode RF FEM using Si- and LTCC-based embedded technologies," in IEEE MTT-S Int. Microw. Sym. Dig., 2012, pp. 1-3.

[9] K.-C. Lin, C.-F. Chang, M.-C. Wu, and S.-J. Chung, "Dual-bandpass filters with serial configuration using LTCC technology," IEEE Trans. Microw. Theory Techn., vol. 54, no. 6, pp. 2321-2326, Jun. 2006.

[10] S. Oshima, K. Wada, R. Murata, and Y. Shimakata, "Multilayer dualband bandpass filter in low-temperature cofired ceramic substrate for ultra-wideband applications," IEEE Trans. Microw. Theory Techn., vol. 58, no. 3, pp. 614-623, Mar. 2010.

[11] C.-C. Chen, "Dual-band bandpass filter using coupled resonator pairs," IEEE Microw. Wireless Compon. Lett., vol. 15, no. 4, pp. 259-261, Apr. 2005.

[12] K. W. Qian and X. H. Tang, "Compact dual-band semilumped bandpass filter with LTCC technology," Electron. Lett., vol. 47, no. 13, pp. 755-757, Jun. 2011

[13] Y. P. Zhang and M. Sun, "Dual-band microstrip bandpass filter using stepped-impedance resonators with new coupling schemes," IEEE Trans. Microw. Theory Techn., vol. 54, no. 10, pp. 3779-3785, Oct. 2006.

[14] K.-S. Chin, J.-L. Hung, C.-W. Huang, J. S. Fu, B.-G. Chen, and T.-J. Chen, "LTCC dual-band stepped-impedance-stub filter constructed with vertically folded structure," Electron. Lett., vol. 46, no. 23, pp. 1554-1556, Nov. 2010.

[15] M. M. Fahmi, J. A. Ruiz-Cruz, R. R. Mansour, and K. A. Zaki, "Recent results on compact broad-band and multiband low-temperature cofired ceramic components for radio frequency front-ends," IET Microw. Antennas Propag., vol. 5, no. 8, pp. 870-876, Jun. 2011.

[16] B.-J. Chen, T.-M. Shen, and R.-B. Wu, "Dual-band vertically stacked laminated waveguide filter design in LTCC technology," IEEE Trans. Microw. Theory Techn., vol. 57, no. 6, pp. 1554-1562, Jun. 2009.

[17] W.-L. Tsai and R.-B. Wu, "Tri-band filter design using substrate integrated waveguide resonators in LTCC," in IEEE MTT-S Int. Microw. Sym. Dig., 2010, pp. 445-448.

[18] K. Huang, T. Chiu, and H.-B. Wu, "Compact LTCC tri-band filter design," in Proc. Asia-Pac. Microw. Conf., 2007, pp. 1795-1798.

[19] V. Turgaliev, D. Kholodnyak, I. Vendik, D. Stöpel, S. Humbla, J. Müller, and M. A. Hein, "LTCC highly loaded cavities for the design of single- and dual-band low-loss miniature filters," in Proc. Euro. Microw. Conf., 2010, pp. 180-183.

[20] V. Turgaliev, D. Kholodnyak, and I. Vendik, "Small-size dual-band filters on capacitively loaded cavities," in Proc. Euro. Microw. Conf., 2013, pp. 660-663.

[21] L. K. Yeung, K.-L. Wu, and Y. E. Wang, "Low-temperature cofired ceramic LC filters for RF applications," IEEE Microw. Mag., vol. 9, no. 5, pp. 118-128, Oct. 2008. 
[22] Y.-H. Jeng, S.-F. R. Chang, and H.-K. Lin, "A high stopband-rejection LTCC filter with multiple transmission zeros," IEEE Trans. Microw. Theory Techn., vol. 54, no. 2, pp. 633-638, Feb. 2006.

[23] C.-W. Tang and H.-C. Hsu, "Development of multilayered bandpass filters with multiple transmission zeros using open-stub/short-stub/serial semilumped resonators," IEEE Trans. Microw. Theory Techn., vol. 58, no. 3, pp. 624-634, Feb. 2010.

[24] L. K. Yeung and K.-L. Wu, "A compact second-order LTCC bandpass filter with two finite transmission zeros," IEEE Trans. Microw. Theory Techn., vol. 51, no. 2, pp. 337-341, Feb. 2003.

[25] H.-H. Huang and T.-S. Horng, "Capacitive-coupled resonator based bandpass filter with controllable multiple transmission zeros at stopband," Microw. Opt. Technol. Lett., vol. 55, no. 7, pp. 1563-1565, Jul. 2013.

[26] H.-H. Huang and T.-S. Horng, "Design of compact bandpass filter with controllable multiple transmission zeros using the second-order inductive-coupled resonator," Microw. Opt. Technol. Lett., vol. 55, no. 9, pp. 2155-2157, Sep. 2013.

[27] C.-F. Chang and S.-J. Chung, "Bandpass filter of serial configuration with two finite transmission zeros using LTCC technology," IEEE Trans. Microw. Theory Techn., vol. 53, no. 7, pp. 2383-2388, Jul. 2005.

[28] H.-H. Huang, S.-Y. Xu, and T.-S. Horng, "Fast prototype-based design approach to miniaturized LTCC band-pass filters using two reflection zeros," in Proc. Euro. Microw. Conf., 2006, pp. 545-548.

[29] H.-H. Huang and T.-S. Horng, "Fast prototype-based design approach to highly miniaturized LTCC BPFs for WLAN/WiMAX dual-mode applications," Microw. Opt. Technol. Lett., vol. 56, no. 1, pp. 8-11, Jan. 2014.

[30] Y.-C. Lin, H.-H. Huang, and T.-S. Horng, "Synthesis of LTCC multiband bandpass filter using reflection and transmission zeros," in IEEE MTT-S Int. Microw. Sym. Dig., 2014, pp. 1-4.

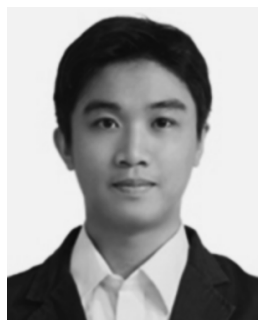

Yi-Chieh Lin (S'11) was born in Kaohsiung, Taiwan, in 1984. He received the B.S.E.E. degree from the National Yunlin University of Science and Technology, Yunlin, Taiwan, in 2006, the M.S.E.E. degree from Chang Gung University, Taoyuan, Taiwan, in 2008, and is currently working toward the $\mathrm{Ph} . \mathrm{D}$. degree in electrical engineering at the National Sun Yat-Sen University, Kaohsiung, Taiwan.

His current research interests include radio frequency (RF) component and IC designs, RF system-in-package, and signal integrity.

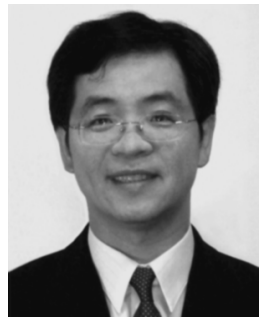

Tzyy-Sheng Horng (S'88-M'92-SM'05) was born in Taichung, Taiwan, on December 7, 1963. He received the B.S.E.E. degree from the National Taiwan University, Taipei, Taiwan, in 1985, and the M.S.E.E. and $\mathrm{Ph} . \mathrm{D}$. degrees from the University of California at Los Angeles (UCLA), Los Angeles, CA, USA, in 1990 and 1992 , respectively.

Since August 1992, he has been with the Department of Electrical Engineering, National Sun Yat-Sen University, Kaohsiung, Taiwan, where he was the Director of the Telecommunication Research and Development Center (2003-2008), Director of the Institute of Communications Engineering (2004-2007), and is currently a Professor. He has authored or coauthored over 200 technical publications published in refereed journals and conferences proceedings, mostly in IEEE publications. He holds over 30 patents. His research interests include RF and microwave integrated circuits (ICs) and components, RF signal integrity for wireless system-in-package, digitally assisted $\mathrm{RF}$ technologies, and green radios for cognitive sensors and Doppler radars.

Dr. Horng is a member of the IEEE MTT-S Technical Committee MTT-10 and MTT-20. He has served on several Technical Program Committees of international conferences including the International Association of Science and Technology for Development (IASTED) International Conference on Wireless and Optical Communications, the IEEE Region 10 International Technical Conference, the IEEE International Workshop on Electrical Design of Advanced Packaging and Systems (EDAPS), the Asia-Pacific Microwave Conference (APMC), the IEEE Radio and Wireless Symposium (RWS), and the Electronic Components and Technology Conference (ECTC). He has also served on the Project Review Board of the Programs of Communications Engineering and Microelectronics Engineering of the Ministry of Science and Technology, Taiwan. He is the Founder Chair of the IEEE Microwave Theory and Techniques Society (IEEE MTT-S) Tainan Chapter. He is currently an Associate Editor for the IEEE TRANSACTIONS ON MiCROWAVE THEORY AND TECHNIQUES. He was the recipient of the 1996 Young Scientist Award presented by the International Union of Radio Science, the 1998 Industry-Education Cooperation Award presented by the Ministry of Education, Taiwan, and the 2010 Distinguished Electrical Engineer Award presented by the Chinese Institute of Electrical Engineering, Kaohsiung Branch, Taiwan. Recently, he was the recipient of the 2011 Advanced Semiconductor Engineering (ASE) Inc. Chair Professorship and the 2012 Outstanding Research Award of National Sun Yat-Sen University.

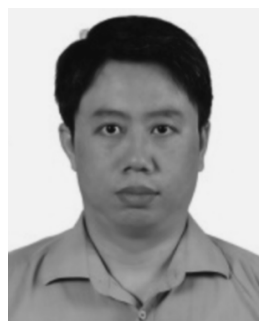

Hui-Hsiang Huang was born in Pingtung, Taiwan, in 1972. He received the Ph.D. degree in electrical engineering from the National Sun Yat-Sen University, Kaohsiung, Taiwan, in 2013.

From 2004 to 2011, he was with the Chilisin Electronics Inc., Hsinchu, Taiwan, as a Research Deputy Manager in charge of the electrical design and modeling of low-temperature cofired ceramic (LTCC) components. In 2011, he joined Infomax Communication Company Ltd., Hsinchu, Taiwan, where he is a Project Manager of the mixed-signal IC group for developing power and signal integrity cosimulation of IC, package and PCB, and the evaluation board design of smart phone. 